\title{
Analysis of morphological changes as a key method in studying psychiatric animal models
}

\author{
Oliver von Bohlen und Halbach
}

Received: 27 November 2012 / Accepted: 5 December 2012 /Published online: 20 January 2013

(C) The Author(s) 2013. This article is published with open access at Springerlink.com

\begin{abstract}
A major interest in the analysis of animal models of psychiatric diseases is their underlying cellular pathology and to gain information regarding whether pharmacological treatments, genetic differences or an altered environment exert an impact upon the brain morphology or on the morphology or activity of single neurones. In this review, several key methods will be introduced that allow the analysis of morphological changes that are frequently observed in psychiatric animal models. An overview of the techniques that enable dendritic arborisation, alterations in dendritic spines and changes in fibre densities to be analysed are described. Moreover, methods for the analysis of adult neurogenesis and neurodegeneration and for the analysis of neuronal activity in fixed brain tissue are described. An important step during the analysis of morphological changes is the estimation of the number of stained cells. Since conventional cell counting methods have several limitations, two different approaches that permit an estimate of the number of stained cells within three-dimensional tissue are also discussed.
\end{abstract}

Keywords Dendritic spines · Sholl analysis $\cdot c$-fos · Stereology $\cdot$ Morphometry $\cdot$ Optical disector $\cdot$ Abercrombie . Correction

\section{Introduction}

A major interest in the analysis of animal models of psychiatric diseases is to gain information regarding whether

The work of the author is supported by the DFG (BO 1971/5-1 and BO 1971/6-1).

O. von Bohlen und Halbach $(\bowtie)$

Institut für Anatomie und Zellbiologie, Ernst-Moritz-Arndt-

Universität Greifswald, Friedrich-Loeffler-Strasse 23c,

17487 Greifswald, Germany

e-mail: oliver.vonbohlen@uni-greifswald.de pharmacological manipulations, treatments, genetic differences or an altered environment exert an impact upon the behaviour of the animals. If such differences can be found, knowledge of whether these changes are accompanied, on the one hand, by changes in the gross morphology or, on the other, by changes in the electrophysiological properties of certain neuronal populations is of interest. Changes in gross morphology can be analysed by using magnetic resonance imaging (MRI), whereas changes in functional properties can be examined in detail by using electrophysiological approaches.

In addition, changes can also occur at the morphological level, ranging from alterations in the total number of neurones in a brain area (e.g., often seen in the case of models of neurodegenerative diseases) to changes in the number of neuronal subpopulations. For example, adult neurogenesis in the hippocampus has gathered much attraction, since functional neurogenesis in this subregion has been discovered to have an impact upon certain forms of learning and memory formation. Moreover, adult neurogenesis might have an impact upon depression, since several antidepressants have been discovered that can increase the rate of adult neurogenesis within the hippocampus (Danzer 2012). Changes in the morphology of individual neurones can occur, including (to name just a few) altered dendritic arborisation and changes in dendritic spine densities or length of individual spines. The analysis of such morphological changes is essential to understanding the mechanisms that can translate them into behavioural changes in these animal models.

Functional magnetic resonance imaging (fMRI) can be used to study changes in blood flow or flow-related phenomena in human subjects in vivo (Jueptner and Weiller 1995). The fMRI technique is based on the difference in the magnetic properties of oxyhaemoglobin, which is the oxygen carrier and of deoxyhaemoglobin, which is a product 
produced in the areas of oxygen consumption, i.e., the brain parenchyma. This ratio is reflected as the physical phenomenon BOLD (blood oxygenation level dependent), which is thought to represent a marker of neuronal activity (Shtark et al. 2012). However, despite being an elegant method to examine the brain areas that show neuronal activity, the resolution offered by fMRI might not be high enough to analyse neuronal activity at the cellular level. Changes in neuronal activity at the morphological level can, for example, be evaluated by analysing the expression of "activity markers", such as c-fos. The counting of cells that are positive for an "activity marker" directly and a comparison of these numbers with those obtained by direct counting of the labelled cells under control conditions seem to represent a straightforward method for obtaining meaningful data. However, since a brain section represents a threedimensional space, the direct counting of labelled cells has several disadvantages.

In this review, various methods are introduced that allow a detailed analysis of those morphological changes that are related to behavioural alterations in animal models of psychiatric disorders. These methods include the analysis of dendritic branching patterns and the analysis of dendritic spines and fibre densities. Moreover, methods for analysing adult neurogenesis are discussed, as is the use of markers for analysing neuronal activity at the cellular level. Since the counting of cells in a three-dimensional tissue is much more complicated than counting cell numbers in a twodimensional environment, e.g., in cell culture dishes, a section of this review is devoted to the various methods of estimating neuronal densities or total neuronal numbers in brain tissue.

\section{Dendritic arborisation}

Neurones display elongated neuronal trees (axons and dendrites) arising from their cell bodies. The axons send signals to other neurones, whereas the dendrites receive signals from other neurones. The shape and complexity of the neuronal trees of a single neurone affect its connectivity. Changes in the complexity of the dendritic arborisation can alter the plastic network connectivities and thereby induce profound changes in neuronal networks and neuronal plasticity. To obtain insights into possible changes in the morphology of individual neurones, a method that allows the quantitative analysis of the relevant morphological characteristics is required. A widely used method is the so-called Sholl analysis, which was initially described in 1953 (Sholl 1953). The Sholl analysis enables us to describe the distribution of neuronal segments at various distances away from the cell body. Three main methods of the Sholl analysis are in use, namely the linear, semi-log and loglog methods (Sholl 1953; Milosevic and Ristanovic 2007), whereby the linear method is the method that is mainly used. Quantification can be undertaken by reconstructing a neurone and superimposing a series of concentric circles (spheres in three dimensions) of gradually increasing radius around the soma of the neurone (Fig. 1a). Within each of the rings or spheres, various metrics can be obtained, such as the number of intersecting dendrites, the number of branching points ("intersection number") and the length of the intersecting dendrites. Furthermore, the total length of each dendrite can be calculated, as can the order of the dendritic branch. From these data, we can also make graphical representations of a neurone (Fig. 1b). The greater the number of concentric circles and the smaller the intercircle intervals, the more precise is the analysis (Gutierrez and Davies 2007).

The Sholl method has been used, for example, to analyse dendritic alterations in the cortex of humans suffering from Rett syndrome (Armstrong et al. 1995) and in a mouse model of amyotrophic lateral sclerosis (Sgobio et al. 2008). By using the Sholl analysis, changes in dendritic complexity caused by prenatal stress have been detected
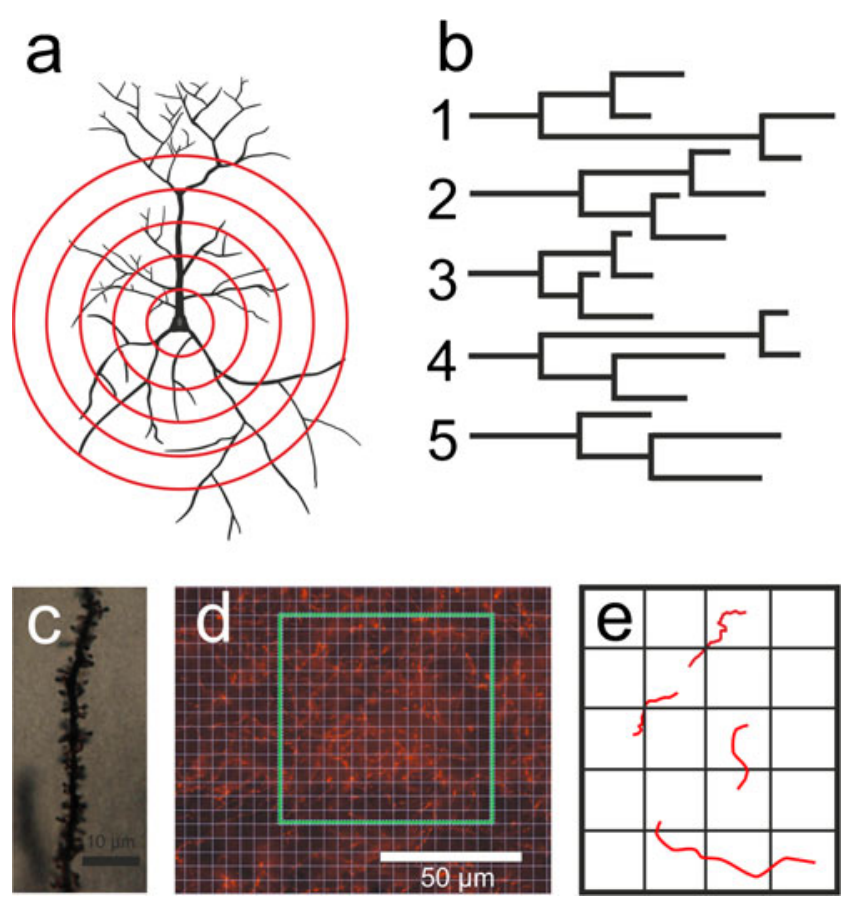

Fig. 1 a Representation of the Sholl analysis. Quantification can be made by superimposing a series of concentric circles of gradually increasing radius around the soma of the neurone. Branching points, intersections and endings can be calculated with this method. b Example of a graphical representation of the dendritic branching pattern of a neurone. $\mathbf{c}$ Dendrite of a cortical pyramidal neurone with its dendritic spines (Golgi-Cox method). d One possible way of analysing the relative fibre densities is to count the number of fibre fragments that intercept grid-points within a counting frame. e Two fibres intercept grid-points (upper left). Since the analysis is conducted on two dimensional images, we cannot determine whether these fibre fragments belong to the same fibre or to different fibres 
(Murmu et al. 2006; Martinez-Tellez et al. 2009) and differences in dendritic morphology have been shown to correlate with anxiety-like behaviour (Miller et al. 2012). In most of these studies, Golgi-impregnated material has been used (Sholl 1953; Schoenen 1982; Sgobio et al. 2008; Martinez-Tellez et al. 2009). However, single neurones that have been filled with neuronal tracers (Miller et al. 2012) can also be used in such analyses.

Nevertheless, the Sholl analysis is only infrequently used, since this technique suffers from the drawback of being an extremely time-consuming method, as it involves gathering a complete set of neurite intersection points in a series of concentric circles centred on the cell soma (Gutierrez and Davies 2007). Thus, the manual drawing and counting of ring intersections in order to generate a Sholl profile takes a great deal of time. To overcome this drawback, computer-assisted methods have been developed. However, limitations still arise, since several computer-assisted protocols require extensive image editing before axons or dendritic trees become clearly distinguishable from the background (Gutierrez and Davies 2007). Gutierrez and Davies (2007) have introduced a Sholl analysis technique that does not require image editing, although this technique requires significant user input to identify axon terminals and bifurcation points for a given neurone. Nonetheless, this method has been used in cases of dissociated neuronal cultures. A further method that allows a semiautomated analysis was introduced in 2010. Compared with conventional manual quantification, the semi-automated method should be about three times faster but with a similar level of sensitivity and minimal inter-user variability (Gensel et al. 2010). However, these last two methods have only been tested in a two-dimensional environment (cell cultures), whereas neurones within brain tissue are situated in a threedimensional space and, therefore, the dendrites cannot be described sufficiently by merely analysing them along the $\mathrm{x}$ and $y$-axes. For the determination of the shape of neurones within the brain, we also need to acquire information concerning the z-axis. Thus, to obtain the full information of the dendrites within a brain section, a Sholl-analysis has to be performed in a three-dimensional space. This requires that the neurones are manually traced or that the tracing is assisted by a computer, which collects, during the reconstruction, the information needed. These computer-guided or computer-aided methods are implanted in various software programs, such as Neurolucida (MBFBioscience) or Imaris (Bitplane Scientific Software).

\section{Dendritic spines}

The dendrites of principal neurones of most brain areas are covered by small protrusions, the so-called dendritic spines. A classical dendritic spine consists in a head that is connected to the dendritic shaft by a narrow neck. Dendritic spines are the main sites of synaptic input for neurones (von Bohlen und Halbach 2009) and act as subcellular compartments that locally control signalling mechanisms. They are crucially involved in receiving and processing synaptic information. Spine density is related to the amount of connectivity between the neurones with dendritic spines and those axons from other neurones that build up synaptic contacts. One role of dendritic spines is therefore the establishment and the maintenance of these connections. Furthermore, dendritic spines also seem to be involved in further functions, since they compartmentalise calcium and other signalling components that are involved in synaptic efficacy (Nimchinsky et al. 2002).

Dendritic spines are thought to play a prominent role in neuronal plasticity and several forms of learning have been shown to increase the number of dendritic spines (Geinisman 2000; Leuner and Shors 2004). For example, spatial training of adult rats has been demonstrated to increase the learning ability associated with a significant increase in the spine densities of CA1 pyramidal neurones. These experiments have led to the conclusion that such an increase in spine density is responsible for altered connectivity, as a result of spatial training (Moser et al. 1994). Likewise, during aging, the decline in memory and cognitive capacities is accompanied by a reduction in the spine densities of CA1 pyramidal neurones (von Bohlen und Halbach et al. 2006b).

Alterations in dendritic spines have been found under a variety of conditions, including not only genetic disorders that result in mental retardation (von Bohlen und Halbach 2010a), such as Down's and fragile-X syndromes but also cases of traumatic lesions, progressive neurodegeneration and alcohol or toxin exposure (Fiala et al. 2002). Changes in dendritic spines have further been noted in cases of schizophrenia (Bennett 2011) and major depression (Law et al. 2004). Mainly reductions in spine densities have been described in major depression. Interestingly, treatment with several antidepressants has been shown to produce significant increases in dendritic spine density in an animal model of depression (Norrholm and Ouimet 2001).

The visualisation of dendritic spines mainly involves silverimpregnation methods (so-called Golgi-impregnations). These methods were developed many years ago and were already used by Santiago Ramon y Cajal for visualising and describing the fine structure of the nervous system (see Garcia-Lopez et al. 2007); however, these techniques are still used today, since they allow the visualisation of dendritic spines (Fig. 1c) in high quality. Nevertheless, to obtain good results, only Golgiimpregnated sections that are uniformly dark throughout the section should be used (Leuner et al. 2003) and only dendrites that display no breaks in their staining and that are not obscured by other neurones or artefacts (Liu et al. 2001) should be evaluated. Since primary dendrites are thick and often 
display only a small number of spines, a restricted analysis of spines located on secondary or tertiary dendritic trees might be useful. The number of dendritic spines per length, the so-called "spine density", is mainly calculated by dividing the number of spines on a segment by the length of the segment, the data being expressed as the number of spines per $10 \mu \mathrm{m}$ dendritic length (Leuner et al. 2003; von Bohlen und Halbach et al. 2006a). Such an evaluation can easily be performed by using cell culture systems; however, in brain sections, the dendrites extend through a three-dimensional space. Thus, in order to obtain results that are not biased by the z-axis, individual dendrites have to be traced three-dimensionally at high magnification (usually between $400 \times$ and $1000 \times$ ). These reconstructions can, for example, be made by generating virtual serial sections (in the z-axis) by using a computer-aided system. Such z-stacks can subsequently be used for the reconstruction of the dendrites and their spines by means of, for example, an appropriate computer analysis system. The reconstructions (given that they have been generated at high magnification: $630 \times$ or $1000 \times$ ) also enable the length of the individual dendritic spines to be measured. However, even when reconstructions are employed, the spine density estimates based only on visible spine counts represent an underestimate of the total number of spines. The number of spines invisible to the counter increase with increasing diameters of the dendrites analysed (Horner and Arbuthnott 1991). A basic protocol that describes step-by-step the methodology for the impregnation and staining of neuronal tissue and the quantitative characterisation of neuronal morphology is not provided here, since it has been described recently by Milatovic and coworkers (2010).

\section{Fibre densities}

Since a variety of mental and affective disorders and neurodegenerative diseases are associated with changes in diverse transmitter systems (Nikolaus et al. 2009; Schliebs and Arendt 2011), not only the morphology of dendrites and dendritic spines but also the densities of fibres might be affected in several animal models of psychiatric diseases. Neurotransmitter-bearing fibres are mainly not visualised by antibodies directed against the neurotransmitter itself but by antibodies directed against enzymes that are involved in the formation of the neurotransmitter, such as tyrosine hydroxylase or choline acetyltransferase. In order to avoid the counting of all stained fibres within a brain region, the determination of the relative fibre densities allows a quick and reliable measurement of fibre densities. This method for analysing fibre densities is also suitable for quantifying degenerating axons, e.g., in animal models of epilepsy (von Bohlen und Halbach et al. 2004). In most cases, images are taken at high magnification by using a digital camera and are analysed off-line as follows:

a) a sampling line of $100 \mu \mathrm{m}$ is randomly placed over the image and the number of positive fibres crossing the line is counted in different sections of each brain region and animal (Greferath et al. 2012)

b) a frame with a defined region of interest is randomly placed over the image. In addition, a grid with a lattice spacing of several micrometres (in the $\mathrm{x}, \mathrm{y}$ plane) is also placed over the image. Fibres intercepting the grid points are counted (Fig. 1d) and expressed per unit area of the region analysed. Relative fibre densities are expressed as $\mathrm{Q}=\mathrm{Gi} / \mathrm{Go}$ where $\mathrm{Gi}$ is the grid points intercepted by the fibres and Go is the total number of grid points (von Bohlen und Halbach et al. 2005). However, since fibre densities can be high and since the images might have strong background staining, images can be contrast-enhanced, thresholded and converted to binary images (Mamounas et al. 2000). It should be kept in mind that the fibres run through a three-dimensional space and that, by using this method, only fibres within a single plane are used for this estimate. If two fibre fragments intercept grid-points, we cannot distinguish whether these two fibre fragments belong to a single fibre or whether they belong to two different fibres (Fig. 1e).

\section{Markers for neurogenesis}

Adult neurogenesis can mainly be observed in two brain regions:

- the subventricular zone (SVZ): the newly born cells of the SVZ migrate and differentiate into neurones within the olfactory bulb where they establish synaptic contacts and functional connections with neighbouring cells

- the subgranular zone of the dentate gyrus (DG) of the hippocampus: the newly formed cells integrate into the granular layer of the DG and start to extend their axons and dendrites into their target areas. The later form of adult neurogenesis is also termed "adult hippocampal neurogenesis".

Neurogenesis within the adult brain is not a simple switch from a dividing precursor to a functional mature neurone; instead, adult neurogenesis is a multi-step process (proliferation, differentiation, migration, targeting and synaptic integration) that ends with the formation of a post-mitotic functionally integrated new neurone. Various markers are expressed during specific stages of adult neurogenesis. The availability of such markers allows the time-course and fate 
of newly born cells to be followed in a detailed and precise fashion. Several of the available markers are markers for proliferative events, whereas others are specific for the different phases of neurogenesis within the adult DG (von Bohlen und Halbach 2011). Neurogenesis within the DG occurs throughout postnatal life and is influenced by aging, behaviour and environment (Kempermann et al. 1997; Kim et al. 2002; Uda et al. 2006; von Bohlen und Halbach 2010b). Adult hippocampal neurogenesis has attracted attention and interest, because, as has become increasingly clear, the new neurones exert critical functions in the hippocampus related to particular aspects of learning and memory (Kempermann 2011). The amount of neurogenesis in the DG can be modulated by various factors that increase or decrease the number of newly generated neurones that become incorporated into the circuit; physical exercise or an enriched environment can increase neurogenesis, whereas aging can decrease neurogenesis (Marin-Burgin and Schinder 2012). Currently, several molecular mechanisms that regulate adult neurogenesis have been identified, including intracellular and extracellular players, such as cell cycle regulators, transcription factors and epigenetic regulators (Ming and Song 2011). Moreover, a variety of neurological disease-risk genes have been shown to alter adult neurogenesis, including genes related to Alzheimer's disease, mental retardation and Rett syndrome (Ming and Song 2011). Neurogenesis is down-regulated by stressful stimuli, such as sleep deprivation, social isolation, subordination to a dominant animal or exposure to predator odour (Danzer 2012). Depression has a profound impact upon several brain structures, such as the hippocampal formation. In numerous MRI studies, the hippocampal volumetric differences between depressed and healthy subjects have been analysed. Although some inconsistencies exist among the different studies, a smaller hippocampal volume in depressed patients seems to be related to the pathophysiology of the disease (Eker and Gonul 2010). An interesting aspect of antidepressant treatment is that it is capable of blocking or reversing the hippocampal atrophy that is observed in patients with depression (Schmidt and Duman 2007). As has also been reported, stress paradigms and some animal models of depression result in a decrease in hippocampal volume and neurogenesis and chronic antidepressant treatment is not only effective in the treatment of depression but also upregulates adult hippocampal neurogenesis (Dranovsky and Hen 2006; Gass and Henn 2009; Dokter and von Bohlen und Halbach 2012); these changes are paralleled by behavioural changes in animal models of depression (Dranovsky and Hen 2006).

To analyse possible alterations in neurogenesis in different animal models, one should keep in mind that adult neurogenesis is a multi-step process. Thus, interesting aspects might include not only whether neurogenesis is altered in general but also which steps of neurogenesis are mainly affected (e.g., proliferation or differentiation). By chance, different markers are expressed during specific stages of adult neurogenesis (Fig. 2a). The availability of markers for proliferation and for early and late phases of adult neurogenesis allows the examination of these various steps in detail.

Bromodeoxyuridine (BrdU) is an S-phase-specific marker, which is incorporated into DNA. The visualisation of BrdU by using specific antibodies allows the detection of newly formed cells. BrdU application is mainly conducted by intraperitoneal injection. Depending on the application mode (duration, concentration of applied BrdU and survival times after BrdU injection), the number of labelled cells can vary within the brain (Taupin 2007a, 2007b). Since BrdU is not intrinsically expressed but must be applied, BrdU can be used for "birth dating" and the monitoring of cell proliferation. However, BrdU is not specific for neurogenesis, since it is a general marker of proliferative events. Thus, BrdU labelling does not allow us to distinguish between newly formed glia cells, neuronal cells or other proliferating cells. To determine that changes in BrdU labelling are indeed related to altered neurogenesis, one has to combine this labelling with other markers that label newly formed neurones at later stages in the time-course of neurogenesis (von Bohlen und Halbach 2007). Detailed information concerning the use of BrdU immunohistochemistry for studying adult neurogenesis can be found in the excellent review article by Taupin (2007a).

Antibodies directed against Ki-67, phosphohistone H3 (Fig. 2b), proliferating cell nuclear antigen (PCNA) or minichromosome maintenance protein 2 can be used to stain proliferating cells. These markers are widely used to identify proliferating and mitotic cells in the hippocampus and, in contrast to BrdU, they are intrinsically expressed (von Bohlen und Halbach 2011). Markers, such as glial fibrillary acidic protein, musashi-1, nestin, paired box gene 6 or SRYrelated HMG-box gene 2, can be used to monitor early stages of adult gliogenesis and neurogenesis, as these markers are expressed at early stages of adult neurogenesis. However, whether these cells will become neurones or glia cannot be distinguished. Markers for the neuronal lineage, such as T-box brain gene 2 and the basic helix-loop-helix protein NeuroD, are expressed in mitotically active young neuronal cells, whereas the polysialylated embryonic form of the neural cell adhesion molecule (PSA-NCAM) and doublecortin (DCX) are expressed by both young mitotically active and young postmitotic neurones. Thus, a transient co-expression of PSA-NCAM or DCX occurs (Fig. 2c) with NeuroD and a transient co-expression of PSA-NCAM or DCX occurs with NeuN, a marker for mature neuronal cells, indicating that PSA-NCAM and DCX are markers for the neuronal lineage in the adult DG (von Bohlen und Halbach 2011). The use of antibodies directed against these markers, 

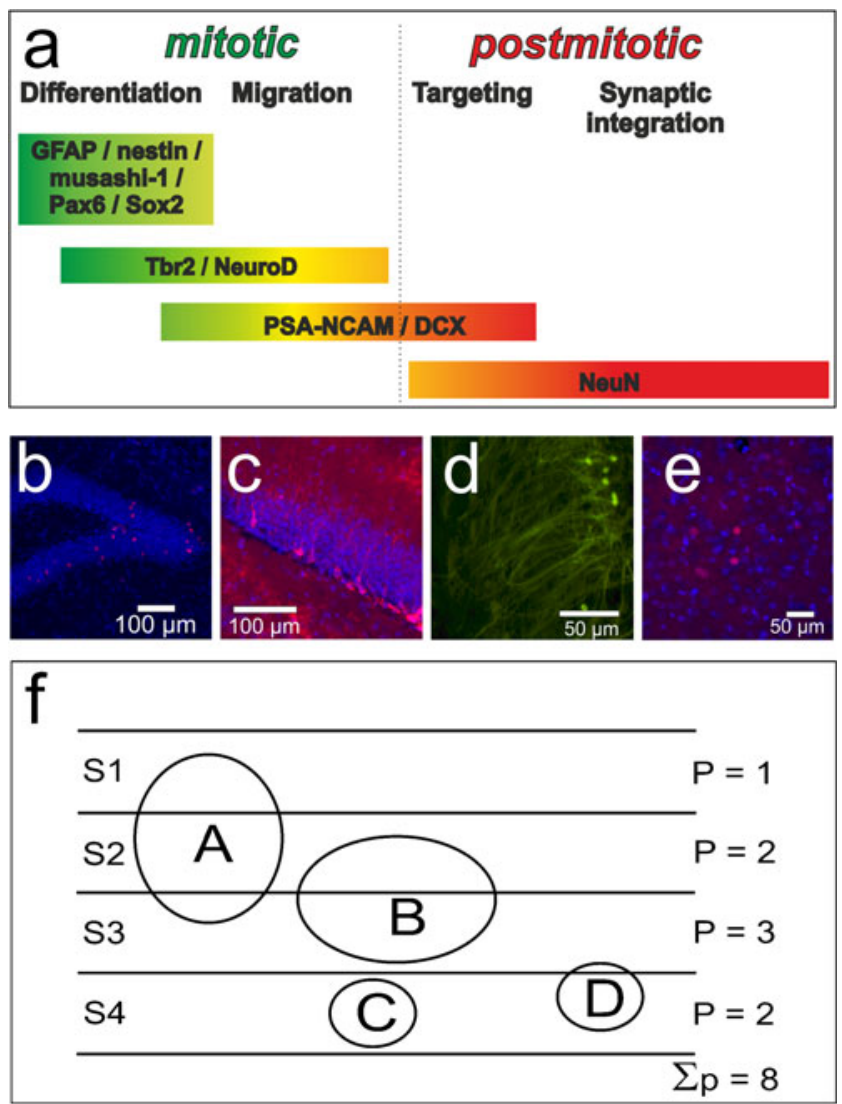

Fig. 2 a Markers suitable for staging adult neurogenesis. The different markers are specific for certain stages of adult neurogenesis and allow the identification not only of proliferating cells but also of young neurones (GFAP glial fibrillary acidic protein, Pax6 paired box gene 6, Sox2 SRY-related HMG-box gene 2, Tbr2 T-box brain gene 2, NeuroD basic helix-loop-helix protein, PSA-NCAM polysialylated embryonic form of the neural cell adhesion molecule, $D C X$ doublecortin, NeuN marker for mature neuronal cells). b Phosphohistone-H3positive cells in the murine dentate gyrus (DG) are shown in red. DAPI (4,6-diamidino-2-phenylindole) was used for visualisation of cell nuclei (blue). c DCX-positive cells in the murine DG. DAPI (blue) was used for nuclear counterstaining. d Cells positive for Fluoro-Jade B (a fluorochrome that allows the visualisation of neuronal degeneration) within the murine brain. e Cells positive for c-Fos (an inducible transcription factor; red) within the basolateral nucleus of the rat amygdala. DAPI (blue) was used for nuclear counterstaining. f Four objects $(A-D)$ are located within a three-dimensional tissue. The four sections $(S 1-S 4)$ through the tissue are indicated by horizontal lines and the objects are represented as circles. Objects $A$ and $B$ are of the same size, as are $C$ and $D$. Objects $A$ and $B$ have a greater height than the section thickness, whereas objects $C$ and $D$ have a smaller height than the section thickness. Because of the size of the objects ("particles") and their distribution within the tissue, the particles are cut and thus the sum of the number of profiles $(P)$ counted is not equivalent to the total number of particles

either alone or in combination, allow us to monitor adult neurogenesis and to examine whether changes in adult neurogenesis are attributable to proliferative events or to differentiation. In order to identify whether significant changes in the population of neurogenic cells occur between two groups of animals, the number of stained cells has to be determined. Several methods for estimating cell numbers or cell densities are presented below.

\section{Neurodegeneration}

Neurodegenerative events can be detected in several disorders of the central nervous system. In particular, epilepsy can induce neurodegeneration. In order to monitor neurodegeneration, several techniques are available. The visualisation of degenerated fibres can be performed by using selective silver impregnations, such as the Nauta-Gygax method (Nauta and Gygax 1954) and the densities of degenerated fibres can be estimated (see above). Cell death, such as apoptotic cell death, can be monitored by using TUNEL or immunohistochemistry for activated caspase-3. However, these protocols are not suitable for visualising only degenerative neurones. In 1997, a fluorochrome that allows the visualisation of neuronal degeneration was introduced (Schmued et al. 1997). This fluorochrome was termed "Fluoro-Jade". Fluoro-Jade-stained neurones display a greenish fluorescent colour. Fluoro-Jade not only allows the detection of the soma of degenerative neurones but also allows the visualisation of their dendritic arborisations (Schmued et al. 1997). Some years later, Fluoro-Jade B (Fig. 2d) and FluoroJade C were introduced (Schmued and Hopkins 2000; Schmued et al. 2005). Both substances have higher affinities than Fluoro-Jade for the entire degenerating neurone, including the cell body, dendrites and axons, whereby Fluoro-Jade C exhibits the greatest signal to background ratio and the highest resolution (Schmued et al. 2005). Fluoro-Jade has successfully been used to analyse neurodegeneration, for example, in animal models of depression (olfactory bulbectomy, Jarosik et al. 2007) or epilepsy (such as pilocarpin treatment, Wang et al. 2008; kindling, Cole-Edwards et al. 2006).

\section{Activity marker}

Activity-dependent changes are required for the learning and memory of transient experiences. Many of these responses to neuronal activation are believed to be mediated by the de novo synthesis of RNA and proteins (Sun et al. 2007). In particular, the expression of certain immediate-early genes is modulated by neurones in response to their stimulation. Among the first proteins that are up-regulated after stimulation are proteins encoded by genes belonging to the inducible transcription factors (ITFs), such as c-Fos (Fig. 2e), c-Jun or Zif268. The expression of Zif268 (also known as Egr1) is, for example, induced by behavioural stimulation, such as spatial learning (Fordyce et al. 1994) and by the expression of c-Fos and c-Jun (Vann et al. 2000; Teather et al. 2005).

Since these proteins accumulate rapidly after neuronal stimulation, their visualisation, by means of immunohistochemistry, 
allows the detection of single activated neurones. c-Fos has become the most widely used functional anatomical marker of activated neurones, since it is only expressed at low levels in the brain under basal conditions and since it is stereotypically induced in response to several extracellular signals (Kovacs 2008) and by synaptic stimulation (Chaudhuri 1997). Moreover, the detection of c-Fos protein is not complicated and can easily be combined with various markers, such as neuropeptides or neuronal tracers (Kovacs 2008). The use of these activity markers might therefore be extremely helpful in the determination of differences in neuronal activity by comparing either genetically altered animals or animals subjected to different behavioural paradigms with appropriate control animals. The c-Fos method has been used, for example, in analyses of animal models of depression, such as learned helplessness (Enkel et al. 2010) or olfactory bulbectomy (Roche et al. 2007).

Aside from these factors, several other substances have been identified that might function as useful markers for mapping neuronal activity, such as $\mathrm{Cbp} / \mathrm{p} 300$-interacting transactivator with ED-rich tail 2, CCAAT/enhancer-binding protein b, neuronal orphan receptor-1 (Sun et al. 2007) or activity-regulated cytoskeletal-associated protein (Arc). Arc is induced by neuronal excitation and is considered to play a key role in activity-dependent synaptic modification (Lyford et al. 1995). Interesting features of this protein are that the newly-synthesised Arc mRNA is rapidly delivered into active synaptic sites (Steward et al. 1998) and that Arc labels activated dendrites (Kovacs 2008).

However, we should keep in mind that problems occur in the use of ITFs as markers for neuronal activity, since reports have been presented that not all neurones express a particular ITF. Thus, these markers cannot be considered as general markers of neuronal activity for application throughout the central nervous system (Chaudhuri 1997; Kovacs 2008).

Once successful staining has been achieved with an activity marker, one should think of means of analysing these staining patterns. Various modes of analysis are available; thus, in several reports (Vann et al. 2000; Teather et al. 2005; Roche et al. 2007; Dodd et al. 2010), the number of stained profiles were quantified in a defined area and expressed as numbers of immunoreactive cells per section in each brain area or the counts for an area of interest were obtained from several alternate sections to produce a mean for each animal. Hence, different results might be obtained depending on the mode of quantification. The advantages and disadvantages of these (and other) methods for quantification are discussed below.

\section{Cell counts}

After successful staining, the next crucial step is to estimate the number of stained cells and to compare these estimates between different groups. Two different approaches might be of interest: the determination of stained cells within a given two-dimensional area or the determination of the total number of stained cells within a brain region.

In the first case, the total cell number is not determined but rather the number of cells within a region of interest, e.g., within a brain area. By this method, the cell numbers within a two-dimensional area is determined. The data are thought to represent cell densities. However, within a given brain area, the cells might not be uniformly distributed. Therefore, serial sections are required and the cell densities have to be determined in different subsequent sections, e.g., in every third section. Thus, the counts are performed on single histological sections and the data are expressed as mean densities of the counted elements.

Another approach is to use serial sections and to count either every stained cell in a given brain area throughout all sections or to count the cells in, for example, every third section and afterwards to multiply the numbers by three. Using this method, one should obtain an estimate of the total number of stained cells within a brain area. Both methods are easy to perform but they are not comparable, since cell densities do not necessarily allow any conclusions to be drawn concerning total cell numbers.

Unfortunately, these methods have severe limitations, if they are used to determine the number of stained cells in a three-dimensional tissue that is sectioned. First, the observer believes that cells are being counted; however, not cells but their profiles are being counted. A profile is a slice of a particle (in this case a stained cell) that appears in a histological section (Gundersen et al. 1988). If the cell is thicker than the section thickness, several profiles of a single cell can be detected in adjacent sections (Fig. 2f).

If $30 \%$ more labelled neuronal profiles are detected after an experimental manipulation, the usual conclusion would be that the manipulation leads to an increase of $30 \%$ in the number of labelled neurones. Unfortunately, this is rarely the case, because of a lack of a simple relationship between particle and profile numbers (Coggeshall 1992), since the cells might have also changed their shape. A solution might be the use of sections that are thicker than the cells. However, even in this case, one problem still persists: during processing, the tissue shrinks. Thus, measurements should be corrected for this, e.g., by applying a shrinkage factor. For the calculation of this factor, one needs the section thickness after cutting the tissue and the postprocessing thickness, which can be obtained by measuring the tissue thickness in the $\mathrm{z}$-axis after embedding.

A comparison of the profile counts might suggest an increase in cell densities when comparing the experimental with the control situation. However, this might also be attributable to changes in the size of the cells or to changes in the volume of the brain area analysed. Changes in the size of the cells can be detected by measuring their height and 
the volume of a brain area can either be determined by using Cavalieri's method (Gundersen et al. 1988; Cruz-Orive 1999) or by computer-aided devices.

In order to minimise the problems occurring by counting cells in sections derived from a three-dimensional tissue, several approaches have been suggested.

1. One method is the application of a correction formula as introduced by Abercrombie (1946). This formula converts the number of profiles counted within a threedimensional section to an estimate of the true number of objects. The Linderstrøm-Lang/Abercrombie equation is

$\mathrm{N}=\mathrm{n} *(\mathrm{t} /(\mathrm{t}+\mathrm{H}))$ or $\mathrm{N} / \mathrm{n}=\mathrm{t} /(\mathrm{t}+\mathrm{H})$

$\mathrm{N}$ is an estimate of the number of objects whose central points lie within the sampled portions of a defined region in one or multiple sections

$\mathrm{n}$ is the counted number of object segments in the sampled portions of that region in those sections, by using an unbiased sampling design

$\mathrm{t}$ is the (mean) thickness of the section(s) in which $n$ is counted

$\mathrm{H}$ is the mean height of the objects, measured perpendicularly to the plane of section (Hedreen 1998).

This method is unbiased as a thought experiment, regardless of the variation in the object size, shape or anisotropicity but in practice suffers from some difficulties (Hedreen 1998). Abercrombie's method is easy to use and, since many investigators are interested to find out whether a relative and significant difference occurs in numbers between treatment and control groups, rather than attempting to estimate absolute numbers, this method is in most cases sufficient (von Bartheld 2002; Schober et al. 2007). The errors that might be introduced with this method are thought to be negligible when the section thickness exceeds the greatest nuclear height by a factor of about 1.5 (Clarke 1992).

2. Another approach is the use of stereological methods (Gundersen et al. 1988), such as the optical disector or optical fractionator. In the case of the optical disector, a two-step process is required that involves the estimation of both the volume of the structure (est $\mathrm{V}_{\text {ref }}$ ) and the volume density (est $\mathrm{N}_{\mathrm{V}}$ ). The product of the two is an estimate of the total number: est $\mathbf{V}_{\text {ref }} *$ est $\mathbf{N}_{\mathbf{V}}=$ est $\mathbf{N}$ (West 1999). The fractionator approach involves the determination of the number of objects, SumQ ${ }^{-}$, in a known fraction (f) of a structure and multiplication of this number by the reciprocal of the fraction sampled, $1 / \mathrm{f}$ which allows an estimate of $\mathrm{N}$ to be obtained by using the equation $\mathbf{e s t} \mathbf{N}=\mathbf{S u m Q}^{-} * \mathbf{1} / \mathbf{f}$ (West 1999). Advantages of the stereological approaches include that they are theoretically unbiased, that no assumptions have to be made concerning the size, shape or orientation of the particles and that at least the optical disector is highly efficient (von Bartheld 2002). Disadvantages include that these methods require thick sections, that accuracy can be affected by differential shrinkage and compression of sections and that some special equipment, such as a sensitive z-axis encoder, is needed (von Bartheld 2002).

Currently, the above-mentioned methods are thought to represent the methods of choice for either estimating the densities of stained cells within a given area or for estimating the total number of stained cells within a brain region. Detailed protocols that describe step-by-step the methodological determination of cell numbers in a three-dimensional tissue are, for example, provided by Williams and colleagues (2003)

\section{Concluding remarks}

In this review, several key methods have been described that allow the analysis of morphological changes that are frequently observed in psychiatric animal models. These morphological methods, especially in combination with other methods, such as behavioural analysis, electrophysiological studies and the analysis of signalling pathways, to name just a few, are essential for an understanding of the changes occurring in the brain that translate into behavioural changes. A detailed insight into these parameters is also essential for the development of future pharmacological or therapeutical strategies.

Open Access This article is distributed under the terms of the Creative Commons Attribution License, which permits any use, distribution and reproduction in any medium, provided the original author(s) and the source are credited.

\section{References}

Abercrombie M (1946) Estimation of nuclear population from microtome sections. Anat Rec 94:239-247

Armstrong D, Dunn JK, Antalffy B, Trivedi R (1995) Selective dendritic alterations in the cortex of Rett syndrome. J Neuropathol Exp Neurol 54:195-201

Bennett MR (2011) Schizophrenia: susceptibility genes, dendriticspine pathology and gray matter loss. Prog Neurobiol 95:275300

Chaudhuri A (1997) Neural activity mapping with inducible transcription factors. Neuroreport 8:iii-vii

Clarke PG (1992) How inaccurate is the Abercrombie correction factor for cell counts? Trends Neurosci 15:211-212 
Coggeshall RE (1992) A consideration of neural counting methods. Trends Neurosci 15:9-13

Cole-Edwards KK, Musto AE, Bazan NG (2006) c-Jun N-terminal kinase activation responses induced by hippocampal kindling are mediated by reactive astrocytes. J Neurosci 26:8295-8304

Cruz-Orive LM (1999) Precision of Cavalieri sections and slices with local errors. J Microsc 193:182-198

Danzer SC (2012) Depression, stress, epilepsy and adult neurogenesis. Exp Neurol 233:22-32

Dodd GT, Williams SR, Luckman SM (2010) Functional magnetic resonance imaging and c-Fos mapping in rats following a glucoprivic dose of 2-deoxy-D-glucose. J Neurochem 113:1123-1132

Dokter M, von Bohlen und Halbach O (2012) Neurogenesis within the adult hippocampus under physiological conditions and in depression. Neural Regen Res 7:8

Dranovsky A, Hen R (2006) Hippocampal neurogenesis: regulation by stress and antidepressants. Biol Psychiatry 59:1136-1143

Eker C, Gonul AS (2010) Volumetric MRI studies of the hippocampus in major depressive disorder: meanings of inconsistency and directions for future research. World J Biol Psychiatry 11:19-35

Enkel T, Gholizadeh D, von Bohlen und Halbach O, Sanchis-Segura C, Hurlemann R, Spanagel R, Gass P, Vollmayr B (2010) Ambiguouscue interpretation is biased under stress- and depression-like states in rats. Neuropsychopharmacology 35:1008-1015

Fiala JC, Spacek J, Harris KM (2002) Dendritic spine pathology: cause or consequence of neurological disorders? Brain Res Brain Res Rev 39:29-54

Fordyce DE, Bhat RV, Baraban JM, Wehner JM (1994) Genetic and activity-dependent regulation of zif268 expression: association with spatial learning. Hippocampus 4:559-568

Gass P, Henn FA (2009) Is there a role for neurogenesis in depression? Biol Psychiatry 66:3-4

Garcia-Lopez P, Garcia-Marin V, Freire M (2007) The discovery of dendritic spines by Cajal in 1888 and its relevance in the present neuroscience. Prog Neurobiol 83:110-130

Geinisman Y (2000) Structural synaptic modifications associated with hippocampal LTP and behavioral learning. Cereb Cortex 10:952-962

Gensel JC, Schonberg DL, Alexander JK, McTigue DM, Popovich PG (2010) Semi-automated Sholl analysis for quantifying changes in growth and differentiation of neurons and glia. J Neurosci Methods 190:71-79

Greferath U, Trieu J, Barrett GL (2012) The p75 neurotrophin receptor has nonapoptotic antineurotrophic actions in the basal forebrain. $\mathrm{J}$ Neurosci Res 90:278-287

Gundersen HJ, Bendtsen TF, Korbo L, Marcussen N, Moller A, Nielsen K, Nyengaard JR, Pakkenberg B, Sorensen FB, Vesterby A et al (1988) Some new, simple and efficient stereological methods and their use in pathological research and diagnosis. APMIS 96:379-394

Gutierrez H, Davies AM (2007) A fast and accurate procedure for deriving the Sholl profile in quantitative studies of neuronal morphology. J Neurosci Methods 163:24-30

Hedreen JC (1998) Lost caps in histological counting methods. Anat Rec 250:366-372

Horner CH, Arbuthnott E (1991) Methods of estimation of spine densityâ€"are spines evenly distributed throughout the dendritic field? J Anat 177:179-184

Jarosik J, Legutko B, Unsicker K, von Bohlen und Halbach O (2007) Antidepressant-mediated reversal of abnormal behavior and neurodegeneration in mice following olfactory bulbectomy. Exp Neurol 204:20-28

Jueptner M, Weiller C (1995) Review: does measurement of regional cerebral blood flow reflect synaptic activity? Implications for PET and fMRI. Neuroimage 2:148-156

Kempermann G (2011) Seven principles in the regulation of adult neurogenesis. Eur J Neurosci 33:1018-1024
Kempermann G, Kuhn HG, Gage FH (1997) More hippocampal neurons in adult mice living in an enriched environment. Nature 386:493-495

Kim SH, Kim HB, Jang MH, Lim BV, Kim YJ, Kim YP, Kim SS, Kim EH, Kim CJ (2002) Treadmill exercise increases cell proliferation without altering of apoptosis in dentate gyrus of Sprague-Dawley rats. Life Sci 71:1331-1340

Kovacs KJ (2008) Measurement of immediate-early gene activationâ $€$ "c-fos and beyond. J Neuroendocrinol 20:665-672

Law AJ, Weickert CS, Hyde TM, Kleinman JE, Harrison PJ (2004) Reduced spinophilin but not microtubule-associated protein 2 expression in the hippocampal formation in schizophrenia and mood disorders: molecular evidence for a pathology of dendritic spines. Am J Psychiatry 161:1848-1855

Leuner B, Shors TJ (2004) New spines, new memories. Mol Neurobiol 29:117-130

Leuner B, Falduto J, Shors TJ (2003) Associative memory formation increases the observation of dendritic spines in the hippocampus. J Neurosci 23:659-665

Liu WS, Pesold C, Rodriguez MA, Carboni G, Auta J, Lacor P, Larson J, Condie BG, Guidotti A, Costa E (2001) Down-regulation of dendritic spine and glutamic acid decarboxylase 67 expressions in the reelin haploinsufficient heterozygous reeler mouse. Proc Natl Acad Sci USA 98:3477-3482

Lyford GL, Yamagata K, Kaufmann WE, Barnes CA, Sanders LK, Copeland NG, Gilbert DJ, Jenkins NA, Lanahan AA, Worley PF (1995) Arc, a growth factor and activity-regulated gene, encodes a novel cytoskeleton-associated protein that is enriched in neuronal dendrites. Neuron 14:433-445

Mamounas LA, Altar CA, Blue ME, Kaplan DR, Tessarollo L, Lyons WE (2000) BDNF promotes the regenerative sprouting, but not survival, of injured serotonergic axons in the adult rat brain. $\mathrm{J}$ Neurosci 20:771-782

Marin-Burgin A, Schinder AF (2012) Requirement of adult-born neurons for hippocampus-dependent learning. Behav Brain Res 227:391-399

Martinez-Tellez RI, Hernandez-Torres E, Gamboa C, Flores G (2009) Prenatal stress alters spine density and dendritic length of nucleus accumbens and hippocampus neurons in rat offspring. Synapse 63:794-804

Milatovic D, Montine TJ, Zaja-Milatovic S, Madison JL, Bowman AB, Aschner M (2010) Morphometric analysis in neurodegenerative disorders. Curr Protoc Toxicol Chapter 12: Unit 12.16

Miller MM, Morrison JH, McEwen BS (2012) Basal anxiety-like behavior predicts differences in dendritic morphology in the medial prefrontal cortex in two strains of rats. Behav Brain Res 229:280-288

Milosevic NT, Ristanovic D (2007) The Sholl analysis of neuronal cell images: semi-log or log-log method? J Theor Biol 245:130-140

Ming GL, Song H (2011) Adult neurogenesis in the mammalian brain: significant answers and significant questions. Neuron 70:687-702

Moser MB, Trommald M, Andersen P (1994) An increase in dendritic spine density on hippocampal CA1 pyramidal cells following spatial learning in adult rats suggests the formation of new synapses. Proc Natl Acad Sci USA 91:12673-12675

Murmu MS, Salomon S, Biala Y, Weinstock M, Braun K, Bock J (2006) Changes of spine density and dendritic complexity in the prefrontal cortex in offspring of mothers exposed to stress during pregnancy. Eur J Neurosci 24:1477-1487

Nauta WJ, Gygax PA (1954) Silver impregnation of degenerating axons in the central nervous system: a modified technic. Stain Technol 29:91-93

Nikolaus S, Antke C, Muller HW (2009) In vivo imaging of synaptic function in the central nervous system. II. Mental and affective disorders. Behav Brain Res 204:32-66

Nimchinsky EA, Sabatini BL, Svoboda K (2002) Structure and function of dendritic spines. Annu Rev Physiol 64:313-353 
Norrholm SD, Ouimet CC (2001) Altered dendritic spine density in animal models of depression and in response to antidepressant treatment. Synapse 42:151-163

Roche M, Harkin A, Kelly JP (2007) Chronic fluoxetine treatment attenuates stressor-induced changes in temperature, heart rate, and neuronal activation in the olfactory bulbectomized rat. Neuropsychopharmacology 32:1312-1320

Schliebs R, Arendt T (2011) The cholinergic system in aging and neuronal degeneration. Behav Brain Res 221:555-563

Schmidt HD, Duman RS (2007) The role of neurotrophic factors in adult hippocampal neurogenesis, antidepressant treatments and animal models of depressive-like behavior. Behav Pharmacol 18:391-418

Schmued LC, Hopkins KJ (2000) Fluoro-Jade B: a high affinity fluorescent marker for the localization of neuronal degeneration. Brain Res 874:123-130

Schmued LC, Albertson C, Slikker W Jr (1997) Fluoro-Jade: a novel fluorochrome for the sensitive and reliable histochemical localization of neuronal degeneration. Brain Res 751:37-46

Schmued LC, Stowers CC, Scallet AC, Xu L (2005) Fluoro-Jade C results in ultra high resolution and contrast labeling of degenerating neurons. Brain Res 1035:24-31

Schober A, Peterziel H, von Bartheld CS, Simon H, Krieglstein K, Unsicker K (2007) GDNF applied to the MPTP-lesioned nigrostriatal system requires TGF-beta for its neuroprotective action. Neurobiol Dis 25:378-391

Schoenen J (1982) The dendritic organization of the human spinal cord: the dorsal horn. Neuroscience 7:2057-2087

Sgobio C, Trabalza A, Spalloni A, Zona C, Carunchio I, Longone P, Ammassari-Teule M (2008) Abnormal medial prefrontal cortex connectivity and defective fear extinction in the presymptomatic G93A SOD1 mouse model of ALS. Genes Brain Behav 7:427-434

Sholl DA (1953) Dendritic organization in the neurons of the visual and motor cortices of the cat. J Anat 87:387-406

Shtark MB, Korostyshevskaia AM, Rezakova MV, Savelov AA (2012) Functional magnetic resonanse imaging and neuroscience. Usp Fiziol Nauk 43:3-29

Steward O, Wallace CS, Lyford GL, Worley PF (1998) Synaptic activation causes the mRNA for the IEG Arc to localize selectively near activated postsynaptic sites on dendrites. Neuron 21:741-751

Sun W, Choi SH, Park SK, Kim SJ, Noh MR, Kim EH, Kim HJ, Kim $\mathrm{H}$ (2007) Identification and characterization of novel activitydependent transcription factors in rat cortical neurons. J Neurochem 100:269-278

Taupin P (2007a) BrdU immunohistochemistry for studying adult neurogenesis: paradigms, pitfalls, limitations, and validation. Brain Res Rev 53:198-214

Taupin P (2007b) Protocols for studying adult neurogenesis: insights and recent developments. Regen Med 2:51-62
Teather LA, Packard MG, Smith DE, Ellis-Behnke RG, Bazan NG (2005) Differential induction of c-Jun and Fos-like proteins in rat hippocampus and dorsal striatum after training in two water maze tasks. Neurobiol Learn Mem 84:75-84

Uda M, Ishido M, Kami K, Masuhara M (2006) Effects of chronic treadmill running on neurogenesis in the dentate gyrus of the hippocampus of adult rat. Brain Res 1104:64-72

Vann SD, Brown MW, Erichsen JT, Aggleton JP (2000) Fos imaging reveals differential patterns of hippocampal and parahippocampal subfield activation in rats in response to different spatial memory tests. J Neurosci 20:2711-2718

von Bartheld C (2002) Counting particles in tissue sections: choices of methods and importance of calibration to minimize biases. Histol Histopathol 17:639-648

von Bohlen und Halbach O (2007) Immunohistological markers for staging neurogenesis in adult hippocampus. Cell Tissue Res 329:409-420

von Bohlen und Halbach O (2009) Structure and function of dendritic spines within the hippocampus. Ann Anat 191:518-531

von Bohlen und Halbach O (2010a) Dendritic spine abnormalities in mental retardation. Cell Tissue Res 342:317-323

von Bohlen und Halbach O (2010b) Involvement of BDNF in agedependent alterations in the hippocampus. Front Aging Neurosci 2:36

von Bohlen und Halbach O (2011) Immunohistological markers for proliferative events, gliogenesis, and neurogenesis within the adult hippocampus. Cell Tissue Res 345:1-19

von Bohlen und Halbach O, Schulze K, Albrecht D (2004) Amygdalakindling induces alterations in neuronal density and in density of degenerated fibers. Hippocampus 14:311-318

von Bohlen und Halbach O, Schober A, Hertel R, Unsicker K (2005) MPTP treatment impairs tyrosine hydroxylase immunopositive fibers not only in the striatum, but also in the amygdala. Neurodegener Dis 2:44-48

von Bohlen und Halbach O, Krause S, Medina D, Sciarretta C, Minichiello L, Unsicker K (2006a) Regional- and age-dependent reduction in trkB receptor expression in the hippocampus is associated with altered spine morphologies. Biol Psychiatry 59:793-800

von Bohlen und Halbach O, Zacher C, Gass P, Unsicker K (2006b) Age-related alterations in hippocampal spines and deficiencies in spatial memory in mice. J Neurosci Res 83:525-531

Wang L, Liu YH, Huang YG, Chen LW (2008) Time-course of neuronal death in the mouse pilocarpine model of chronic epilepsy using Fluoro-Jade C staining. Brain Res 1241:157-167

West MJ (1999) Stereological methods for estimating the total number of neurons and synapses: issues of precision and bias. Trends Neurosci 22:51-61

Williams RW, Bartheld CS von, Rosen GD (2003) Counting cells in sectioned material: a suite of techniques, tools, and tips. Curr Protoc Neurosci Chapter 1: Unit 1.11 